\title{
Lesão neural hansênica: Papel da neurotrofina NGF e da citocina TNFa
}

\author{
Neural injury in leprosy: Role of neurotrofin NGF and cytokine TNF $\alpha$ \\ Lesión neural hansénica: Papel de la neurotrofina NGF y la citoquina TNF $\alpha$
}

Recebido: 06/05/2021 | Revisado: 10/05/2021 | Aceito: 14/05/2021 | Publicado: 30/05/2021

\author{
Suzana Alves Mundim Carneiro \\ ORCID: https://orcid.org/0000-0002-1788-5664 \\ Centro Universitário de Anápolis - UniEvangélica, Brasil \\ E-mail: suzanamundim1234@gmail.com \\ Isabella Tavares Souza \\ ORCID: https://orcid.org/0000-0002-9388-8947 \\ Centro Universitário de Anápolis - UniEvangélica, Brasil \\ E-mail: itavares531@gmail.com \\ Raika Eduarda Rodrigues da Silva \\ ORCID: https://orcid.org/0000-0001-6579-4145 \\ Centro Universitário de Anápolis - UniEvangélica, Brasil \\ Email: rodriguesraika@icloud.com \\ Emerith Mayra Hungria Pinto \\ ORCID: https://orcid.org/0000-0002-3731-0817 \\ Centro Universitário de Anápolis - UniEvangélica, Brasil \\ Email: emerith0706@hotmail.com \\ Aline de Araújo Freitas \\ ORCID: https://orcid.org/0000-0002-4480-4882 \\ Centro Universitário de Anápolis - UniEvangélica, Brasil \\ Email: alinefreitas2@gmail.com
}

\begin{abstract}
Resumo
Os mecanismos imunopatológicos envolvidos no desenvolvimento de lesão neural hansênica ainda não são completamente esclarecidos. Sendo assim, o objetivo deste trabalho foi realizar uma revisão integrativa a fim de identificar o envolvimento da neurotrofina NGF e da citocina TNF $\alpha$ na patogênese da lesão neural hansênica. Esta revisão foi realizada mediante a busca de artigos nas plataformas indexadoras PubMed, SciELO e Google Acadêmico com os Descritores em Ciências da Saúde (DeCS) "Leprosy Reaction", "Nerve Growth Factor (NGF)", "Tumor Necrosis Factor- alpha (TNF $\alpha$ )", sendo selecionados 28 artigos. Os resultados evidenciaram que durante a reação do tipo 1 há expressão tecidual de IL-2, IFN- $\gamma$, TNF $\alpha$, IL-12 e IL-1 $\beta$. Já a reação do tipo 2 ou ENH tem sido associada com altas concentraçães de TNFa, infiltração neutrofílica e ativação do sistema complemento. Altos níveis de NGF foram observados na forma lepromatosa e baixos níveis na forma tuberculoide da doença, pois nesta há estímulo da expressão de TNF $\alpha$ o que contribui para a evolução de lesões neurais. Parece haver uma interação entre NGF e TNF $\alpha$, uma vez que o NGF induz a expressão endógena de TNF $\alpha$ e esse feedback positivo promove uma sobrevida das células neurais saudáveis.
\end{abstract}

Palavras-chave: Reação hansênica; Fator de crescimento neural (NGF); Fator de necrose tumoral alfa (TNF $\alpha$ ).

\begin{abstract}
The immunopathological mechanisms involved in the development of leprosy neural injury are not yet fully understood. Thus, the objective of this study was to perform an integrative review in order to identify the involvement of neurotrophin NGF and cytokine TNF $\alpha$ in the pathogenesis of neural injury in leprosy reactions. This review was carried out by search for scientific articles in the databases PubMed, SciELO and Google Acadêmico following the Descriptors of Health Science (DeCS) "Leprosy Reaction", "Nerve Growth Factor (NGF)", "Tumor Necrosis Factoralpha (TNF $\alpha$ )", and 28 articles was selected. The results showed that in the type 1 reaction there is tissue expression of IL-2, IFN $\gamma, \mathrm{TNF} \alpha$, IL-12 and IL-1 $\beta$. Whereas the type 2 or ENH reaction has been associated with high TNF $\alpha$ concentrations, neutrophilic infiltration and activation of complement system. High levels of NGF were observed in the lepromatous form of the disease and, low levels, in the tuberculoid form, since this stimulates the expression of $\mathrm{TNF} \alpha$, contributing to the evolution of neural lesions. There seems to be an interaction between NGF and TNF $\alpha$, since NGF induces endogenous expression of TNF $\alpha$ and this positive feedback promotes a survival of healthy neural cells. Keywords: Leprosy reaction; Nerve growth factor (NGF); Tumor necrosis factor- alpha (TNF $\alpha$ ).
\end{abstract}

\section{Resumen}

Los mecanismos imunopatológicos implicados en el desarrollo de la lesión neural por lepra aún no se conocen por completo. Por tanto, el objetivo de este trabajo fue realizar una revisión integradora con el fin de identificar la 
implicación de la neurotrofina NGF y la citoquina TNF $\alpha$ en la patogenia de la lesión neural por lepra. Esta revisión se llevó a cabo mediante la búsqueda de artículos en las plataformas de indexación PubMed, SciELO y Google Scholar con los descriptores de ciencias de la salud (DeCS) "Lepra Reaction", "Nerve Growth Factor (NGF)", "Tumor Necrosis Factor-alpha (TNF $\alpha$ )", obteniendo 28 artículos seleccionados. Los resultados mostraron que durante la reacción de tipo 1 hay expresión tisular de IL-2, IFN $\gamma$, TNF $\alpha$, IL-12 e IL-1 $\beta$. La reacción tipo 2 o ENL, por otro lado, se ha asociado con altas concentraciones de TNF $\alpha$, infiltración neutrofílica y activación del sistema del complemento. Se observaron niveles elevados de NGF en la forma lepromatosa y niveles bajos en la forma tuberculoide de la enfermedad, ya que este estimula la expresión de TNF $\alpha$, lo que contribuye a la evolución de las lesiones neurales. Parece haber una interacción entre NGF y TNF $\alpha$, ya que NGF induce la expresión endógena de TNF $\alpha$ y esta retroalimentación positiva promueve la supervivencia de las células neurales sanas.

Palabras clave: Reacción a la lepra; Factor de crescimento neural (NGF); Factor de necrosis tumoral alfa (TNF $\alpha$ ).

\section{Introdução}

A hanseníase é uma doença crônica causada pelo Mycobacterium leprae (M. leprae) um bacilo intracelular obrigatório que apresenta tropismo por macrófagos de pele e células de Schwann dos nervos periféricos (Lowy L \& Ridley DS, 1954). Atualmente a hanseníase ainda persiste como um problema de saúde pública no Brasil, visto que, dos 202.185 casos novos reportados pela Organização Mundial da Saúde (OMS) em todo o mundo, 27.863 foram notificados no Brasil (WHO, 2020).

É considerada uma doença com baixa patogenicidade, porém, apresenta alta infectividade através das vias aéreas superiores quando há contato com doentes bacilíferos sem tratamento, constituindo assim, sua principal via de transmissão (Souza \& Peres, 2019). A resistência ou susceptibilidade à doença estão relacionadas com a forma de interação entre o bacilo e a habilidade em produzir uma resposta inflamatória eficaz em destruir o bacilo. Os pacientes com hanseníase apresentam amplo espectro de manifestações clínicas dermato-neurológicas representadas por lesões de pele únicas ou múltiplas, que podem surgir em qualquer parte do corpo, com alterações de sensibilidade e de sudorese no local da lesão (Ridley \& Jopling, 1966). Diante disso, o Ministério da Saúde tem traçado estratégias focadas na detecção precoce de casos novos, no exame de contatos e na terapêutica adequada, a fim de prevenir e controlar sequelas físicas e sociais associadas à doença (Ministério da Saúde, 2020).

Para fins de pesquisa, a classificação de Ridley e Jopling de 1966 é a mais utilizada e baseia-se em achados clínicos, baciloscópicos, histopatológicos e imunológicos classificando a hanseníase em dois polos estáveis; tuberculoide (TT) e lepromatoso (LL), além das apresentações instáveis, que são separadas em três subgrupos "borderline": bordeline-tuberculoide (BT), boderline-boderline (BB) bordeline-lepromatoso (BL) (Ridley \& Jopling, 1966). A forma indeterminada (I), que corresponde à forma mais precoce da doença, em que se observa apenas uma inflamação cutânea discreta e manifestações indefinidas, não permitindo sua classificação. Sua evolução depende da resposta imunológica do hospedeiro, podendo evoluir para remissão espontânea ou para qualquer uma das formas clínicas da hanseníase (Lowy L \& Ridley DS, 1954). Um sistema de classificação simplificado para hanseníase baseado no número de lesões de pele foi estabelecido pela OMS para fins operacionais e terapêuticos. Segundo este sistema os pacientes podem ser classificados em paucibacilares (PB) e multibacilares (MB). Os pacientes PB apresentam até 5 lesões de pele, e em geral correspondem as formas TT e BT. Por outro lado, os pacientes MB apresentam mais de 5 lesões de pele, e em geral correspondem as formas BL e LL de Ridley e Jopling. (WHO, 1994).

Episódios imuno-inflamatórios agudos denominados reações hansênicas podem ocorrer antes, durante ou após a terapia, sendo caracterizados por uma resposta imune exagerada do hospedeiro à infecção pelo M. leprae. Fatores que alteram o sistema imunológico do hospedeiro, como a ocorrência de gravidez, vacinação, infecções, estresse físico ou psicológico e o uso de medicamentos, podem desencadear o surgimento de reações hansênicas (Abraçado, Cunha, \& Xavier, 2015).

A ocorrência das reações hansênicas gira em torno de 30 a 50\% dos pacientes diagnosticados com hanseníase, sendo 
mais incidente em áreas com menor nível socioeconômico (Mota, Vilanova, Andrade, Silva, \& Ferreira, 2018). Elas são classificadas em Reação do Tipo I ou Reação Reversa (RR) e em Reação do tipo II ou Eritema Nodoso Hansênico (ENH). A Reação do tipo I, acomete principalmente pacientes BL, BB e BT e é representada por reações locais de hipersensibilidade tardia (DTH) caracterizadas pela infiltração de células T CD4+ e inflamação granulomatosa responsável pelas lesões neurais. Clinicamente, sintomas sistêmicos são ausentes, porém, observa-se um aumento da inflamação das lesões cutâneas préexistentes, tornando-se edematosas, sensíveis, e eritematosas, além de dor, parestesia e perda sensorial como resultado da lesão neural. A Reação do tipo II ocorre preferencialmente em pacientes que apresentam as formas clínicas BL e LL e desenvolvem resposta Th2 associada com presença de imunocomplexos circulantes e depositados nos tecidos. Observa-se o aparecimento de novas lesões de pele eritematosas e nódulos sensíveis na face, extremidades e tronco, além de manifestações sistêmicas como febre, artrite, linfadenopatia, orquite, iridociclite e neurite grave (Geluk, 2018; Khadilkar, Patil, \& Shetty, 2021).

A resposta imune desenvolvida frente ao M. leprae determina o curso e as formas clínicas da doença, portanto, o desfecho da doença, bem como o desenvolvimento de reações hansênicas estão intimamente relacionadas com o padrão de resposta imune desenvolvido. Os pacientes que apresentam reações hansênicas estão mais propensos a apresentar injúria neural associada ao dano tecidual, que é indiscutivelmente a consequência clínica mais importante e grave da hanseníase (Fischer, 2017).

As neurotrofinas são uma família de proteínas que regula diferentes aspectos biológicos do desenvolvimento e da função neural, apresentando uma grande importância na neuroplasticidade. Estudos têm demonstrado uma possível função biológica do fator de crescimento neural (NGF - Nerve Growth fator), um importante protagonista na resposta ao dano neural e na patogênese da hanseníase (T. L. de S. Aarão, de Sousa, Falcão, Falcão, \& Quaresma, 2018). Neste contexto, esse trabalho tem como objetivo realizar uma revisão integrativa sobre o envolvimento da neurotrofina NGF e da citocina TNF $\alpha$ na patogênese da lesão neural observada durante a ocorrência das reações hansênicas tipo 1 e 2 .

\section{Metodologia}

Trata-se de uma Revisão Integrativa de literatura, que permite um apanhado e análise de estudos publicados, possibilitando conclusões a respeito de uma determinada área específica (Pereira et al. 2018). O presente estudo determinou como pergunta norteadora: "Qual é a relação da neurotrofina NGF e da citocina TNF alfa com a ocorrência de reações hansênicas e lesão neural?". Foi realizado um levantamento bibliográfico nas bases de dados PubMed, SciELO e Google Acadêmico, o qual permitiu a seleção de 28 artigos científicos publicados entre os anos de 2015 e 2020.

Foram utilizados, para a busca dos artigos, os seguintes descritores em Ciências da Saúde (DeCS): "Leprosy Reaction", "Nerve Growth Factor (NGF)", "Tumor Necrosis Factor- alpha (TNFa)" e seus correspondentes na língua portuguesa. Tais termos foram combinados entre si e associados ao operador booleano OR. Os critérios de inclusão definidos para a seleção dos artigos foram: artigos publicados em português, inglês e espanhol; que contemplem a pergunta norteadora do estudo; e que foram publicados nos referidos bancos de dados nos últimos cinco anos.

Trata-se de uma abordagem quantitativa realizada por três pesquisadores que inicialmente efetuaram uma leitura do título e resumo e, posteriormente, uma leitura na íntegra para selecionar os estudos que contemplaram a temática abordada.

\section{Resultados e Discussão}

O comprometimento dos nervos periféricos é a principal consequência da hanseníase e está presente em todas as suas formas clínicas, desde a mais inicial, como a indeterminada, até as formas polares, como tuberculoide e lepromatosa. Sua fisiopatologia é obscura, devido às limitações apresentadas pelo modelo hansênico experimental, e varia de acordo com a forma clínica que o paciente apresenta (Khadilkar et al., 2021). Na neuropatia associada a hanseníase, a presença do bacilo nos 
nervos periféricos e células de Schwann induzem uma resposta imune mediada por macrófagos e outras células que eventualmente são responsáveis pelo aparecimento de lesões imuno-mediadas. Neste contexto, citocinas como TNF $\alpha$, IL-6, e IL-17, podem contribuir para evolução da lesão neural (T. L. S. Aarão, de Sousa, Botelho, Fuzii, \& Quaresma, 2016).

Os dados apresentados por Lopes et al. (2017), relataram que a presença de reação hansênica, seja RR ou ENH, está intimamente relacionada com o dano neural, posto que $78 \%$ dos pacientes que compõe sua amostra apresentaram dano neural e algum tipo de reação hansênica. Destes, não houve prevalência de um tipo de reação sobre o outro, no entanto, houve maior ocorrência de neurite nos casos de pacientes com ENH (Lopes et al., 2017).

A reação do tipo 1 está associada ao aumento abrupto da imunidade celular, devido à participação de linfócitos T e à secreção tecidual de citocinas Th1 (IL-2 e IFN $\gamma$ ) e outras citocinas pró-inflamatórias, como o TNF $\alpha$, IL-12, IL-1 $\beta$ além da quimiocinas MCP-1 (Froes, Trindade, \& Sotto, 2020). Assim sendo, a manutenção da resposta imune celular promove a destruição do bacilo ao mesmo tempo que contribui para intensificação da clínica do paciente, levando a reagudização de lesões antigas e formação de granulomas (Montalvão, L. M; Marques, G. L; Dhamer, D. S. V; Crepaldi, A. A; Sant'ana, A. P; Silva, 2018). A ocorrência de neurites também é frequente e grave, podendo ser a única manifestação clínica apresentada pelo paciente durante a RR. A lesão nervosa na reação do tipo 1, pode resultar de uma neurite silenciosa ou estar associada com espessamento dos nervos periféricos, ocasionando perda de função sensitivo-motora, preferencialmente nos nervos ulnares, medianos, fibular comum, tibial posterior, facial e grande auricular (Santana et al., 2018).

A reação do tipo 2 ou ENH é caracterizada por uma reação inflamatória sistêmica, de evolução rápida, que atinge principalmente pacientes com alta carga bacilar, como os pacientes LL e BL e relaciona-se com deposição de complexos imunes cuja formação pode estar associada com a alta carga bacilar e grande produção de anticorpos, resposta típica do perfil Th2. Sua imunopatogênese tem sido associada com altas concentrações de TNF $\alpha$, infiltração neutrofílica e ativação do sistema complemento, além de alta expressão de IL-17, citocina de assinatura do perfil Th17, o que ocorre paralelamente a uma notável supressão do perfil de células T reguladoras (Froes et al., 2020). Outro estudo demonstrou altos níveis de TGF $\alpha$, IFN $\gamma$ e IL-17 em lesões de pacientes com RT2 quando comparados com lesões de pacientes com RT1 ou na ausência de episódio reacional. Tal estudo também evidenciou aumento de células Treg CD25+ Foxp3+ durante a RT1 o que sugere um possível papel destas células no controle da resposta imune celular exacerbada, a qual pode ser responsável pela lesão neural (M. B. Costa et al., 2018).

Quanto aos níveis circulantes de citocinas, pacientes com ENH apresentam altos níveis de IL-1 e TNF $\alpha$, além do aumento tecidual da expressão de IL-6, IL-8 e IL-10 (P. do S. S. Costa et al., 2018). Já para Morimoto et al. (2017), no ENH há aumento de IFN $\gamma$, TNF $\alpha$, IL-2, IL-6 e queda de IL-10 e IL-4, sendo que o TNF $\alpha$ foi apontado como uma das principais citocinas iniciadoras envolvidas na mediação do dano neural (Morimoto et al., 2017). Níveis aumentados de TNF $\alpha$, TGF- $\beta$ e IL-17 em pacientes com hanseníase e dor neural também foram detectados. Um estudo demonstrou que, na hanseníase, o TNF $\alpha$ e o TGF- $\beta$ induzem apoptose nas células de Schwann, com consequente dano ao nervo periférico (Angst et al., 2020).

Neste contexto, muitos estudos passaram a verificar os níveis de mediadores pró-inflamatórios e pró-regenerativos nas diversas formas clínicas da hanseníase, a fim de identificar se tais mediadores predispõem a lesão neural e consequentemente à incapacidade física. Estudos apontam elevados níveis de IFN $\gamma$ e TNF $\alpha$ plasmático na neuropatia hansênica desencadeada pelas formas clínicas da hanseníase ou durante a ocorrência de reação hansênica, quando comparado ao grupo controle. Dessa forma, altos níveis de IFN $\gamma$ e TNF $\alpha$ parecem funcionar como uma faca de dois gumes, pois, produz o efeito benéfico de eliminação dos bacilos, entretanto, também causa desmielinização e subsequente perda ou comprometimento da função nervosa. Quando se compara as diferentes formas clínicas da hanseníase ou os tipos de reação hansênica, não se observa diferença significativa nos níveis plasmáticos de IFN $\gamma$ e TNFa, sugerindo que a neuropatia na hanseníase envolve ambas as citocinas na mesma extensão embora apresentem diferentes cargas bacilares e manifestações clínicas (Sykam et al., 2015). Entretanto, o TNF $\alpha$ 
parece ter um papel importante na imunopatogênese das reações hansênicas do tipo 1, uma vez que o tratamento preconizado com prednisolona visa reduzir os níveis desta citocina, influenciando assim na resposta imune que ocorre durante o episódio reacional (Oliveira et al., 2016).

A maioria dos estudos têm mostrado que as citocinas pró-inflamatórias apresentam níveis mais elevados durante a ocorrência de reações tipo 1 ou 2, definidas como complicações inflamatórias sistêmicas da hanseníase. Dentre essas citocinas, ressalta-se elevados níveis de TNF $\alpha$ e IL-10, sugerindo que tais citocinas podem predizer a ocorrência de reações do tipo 1 e 2 , respectivamente, enquanto o aumento da IL-1 $\beta$ e IFN $\gamma$ também pode predizer a ocorrência de ambos os tipos reacionais atuando como biomarcadores (Angst et al., 2020).

O NGF é uma neurotrofina endógena que regula aspectos da função neural como desenvolvimento e maturação de células do tecido nervoso. Ao analisar lesões neurais e o epitélio de revestimento da pele, foram observados baixos níveis de NGF e a perda de fibras nociceptivas foi associada a depleção desse fator. Logo, os níveis desse fator e consequentemente a quantidade do seu receptor de alta afinidade (TkrA) se alteram de acordo com as apresentações clínicas da hanseníase, uma vez que altos níveis foram observados na forma lepromatosa e baixos níveis na forma tuberculoide (Cogen et al., 2020). O NGF é descrito como um sinergista de ação redutora do dano tecidual em lesões neurais nas reações hansênicas promovendo regeneração ou apoptose de oligodendrócitos, células de Schwann e neurônios. Por outro lado, essa neurotrofina também atua sobre linfócitos, macrófagos e mastócitos e está intimamente relacionada à indução de inflamação, sendo que, níveis elevados de NGF são frequentemente encontrados em lesões difusas e com comprometimento neural em indivíduos com reações hansênicas. Alguns estudos têm demonstrado que os altos níveis de NGF associados com a forma lepromatosa da doença, estimulam a expressão de TGF $\beta$, que por sua vez reduz o dano tecidual resultante da lesão neural. Além disso, o NGF restaura a sensibilidade neural e exerce efeitos proliferativos e anti-apoptóticos em queratinócitos e células endoteliais (T. L. de S. Aarão et al., 2018).

Por outro lado, baixos níveis de NGF são associados com a forma tuberculoide da doença o que estimula a expressão de TNF $\alpha$ o qual pode contribuir para a evolução de lesões neurais. Baixos níveis de NGF podem também contribuir para o desenvolvimento de neuropatia, bem como perda da sensibilidade, desmielinização neural e degeneração. Desta forma, o NGF pode atuar como um fator protetor para células de Schwann, ou quando em baixos níveis, pode contribuir para o desenvolvimento de neuropatia hansênica (T. L. S. Aarão et al., 2016; de Souza Aarão et al., 2014). Portanto, entende-se que ocorre uma interação entre esses dois fatores em que o NGF induza uma expressão endógena de TNF $\alpha$ e que esse feedback positivo promova uma sobrevida das células neurais saudáveis (T. L. de S. Aarão et al., 2018).

Ainda são escassos os estudos publicados que avaliaram os níveis e a interrelação entre NGF e TNF $\alpha$ durante o episódio reacional, bem como no desencadeamento de lesão neural que ocorre durante as reações, o que demonstra a necessidade da realização de estudos que abordem este tema em específico, haja vista o potencial envolvimento destes dois fatores nos mecanismos imunológicos associados com o dano neural hansênico.

\section{Conclusão}

A citocina TNF $\alpha$ está significativamente aumentada na neuropatia hansênica apresentando um efeito paradoxal, pois, está envolvida nos mecanismos responsáveis pela eliminação dos bacilos, mas em contrapartida pode causar comprometimento da função nervosa. A participação do NGF ainda é indeterminada, podendo atuar como imunomodulador ao proteger células de Schwann ou como fator contribuinte para o desenvolvimento de neuropatia hansênica, quando em baixos níveis. Pouco se sabe acerca do papel do NGF e sua relação com o TNF $\alpha$ no desencadeamento ou prevenção das lesões neurais que ocorrem durante a vigência dos episódios reacionais, o que constitui um entrave na descoberta de possíveis biomarcadores que indiquem predisposição à ocorrência de reações hansênicas, e consequente lesão neural, ou que estejam correlacionados com prevenção 
da lesão ou indução de reparo neural.

Diante de tal fato é imperativo, a realização de estudos que avaliem os níveis plasmáticos e a expressão do NGF e TNF $\alpha$ nas lesões de pele dos pacientes com reação hansênica para elucidar o envolvimento e a interrelação entre NGF e TNF $\alpha$ nos mecanismos que determinam a ocorrência de reações hansênicas, contribuindo assim para que futuramente potenciais marcadores para a predição de lesão neural sejam identificados, ou mesmo para a elaboração de estratégias terapêuticas que interfiram com o mecanismo inibindo o dano neural ou promovendo o reparo do mesmo.

\section{Referências}

Aarão, T. L. de S., de Sousa, J. R., Falcão, A. S. C., Falcão, L. F. M., \& Quaresma, J. A. S. (2018). Nerve growth factor and pathogenesis of leprosy: Review and update. Frontiers in Immunology. https://doi.org/10.3389/fimmu.2018.00939

Aarão, T. L. S., de Sousa, J. R., Botelho, B. S., Fuzii, H. T., \& Quaresma, J. A. S. (2016). Correlation between nerve growth factor and tissue expression of IL17 in leprosy. Microbial Pathogenesis, 90. https://doi.org/10.1016/j.micpath.2015.11.019

Abraçado, M. de F. S., Cunha, M. H. C. M. da., \& Xavier, M. B. (2015). Adesão ao tratamento de hanseníase em pacientes com episódios reacionais hansênicos em uma unidade de referência. Revista Pan-Amazônica de Saúde, 6(2). https://doi.org/10.5123/s2176-62232015000200003

Angst, D. B. M., Pinheiro, R. O., Vieira, J. S. da S., Cobas, R. A., Hacker, M. de A. V. B., Pitta, I. J. R., \& Jardim, M. R. (2020). Cytokine Levels in Neural Pain in Leprosy. Frontiers in Immunology, 11(January), 1-9. https://doi.org/10.3389/fimmu.2020.00023

Cogen, A. L., Lebas, E., de Barros, B., Harnisch, J. P., Faber, W. R., Lockwood, D. N., \& Walker, S. L. (2020). Biologics in leprosy: A systematic review and case report. American Journal of Tropical Medicine and Hygiene, 102(5), 1131-1136. https://doi.org/10.4269/AJTMH.19-0616

Costa, M. B., Hungria, E. M., Freitas, A. A., Sousa, A. L. O. M., Jampietro, J., Soares, F. A., \& Stefani, M. M. A. (2018). In situ T regulatory cells and Th17 cytokines in paired samples of leprosy type 1 and type 2 reactions. PLoS ONE, 13(6), 1-14. https://doi.org/10.1371/journal.pone.0196853

Costa, P. do S. S., Fraga, L. R., Kowalski, T. W., Daxbacher, E. L. R., Schuler-Faccini, L., \& Vianna, F. S. L. (2018). Erythema Nodosum Leprosum: Update and challenges on the treatment of a neglected condition. Acta Tropica. https://doi.org/10.1016/j.actatropica.2018.02.026

de Souza Aarão, T. L., Esteves, N. R., Esteves, N., de Miranda Soares, L. P., da Silva Pinto, D., Fuzii, H. T., \& Quaresma, J. A. S. (2014). Relationship between growth factors and its implication in the pathogenesis of leprosy. Microbial Pathogenesis. https://doi.org/10.1016/j.micpath.2014.10.005

Fischer, M. (2017). Leprosy - an overview of clinical features, diagnosis, and treatment. JDDG - Journal of the German Society of Dermatology, 15(8). https://doi.org/10.1111/ddg.13301

Froes, L. A. R., Trindade, M. A. B., \& Sotto, M. N. (2020). Immunology of leprosy. International Reviews of Immunology, 0(0), 1-21. https://doi.org/10.1080/08830185.2020.1851370

Geluk, A. (2018). Correlates of immune exacerbations in leprosy. Seminars in Immunology. https://doi.org/10.1016/j.smim.2018.06.003

Khadilkar, S. V., Patil, S. B., \& Shetty, V. P. (2021). Neuropathies of leprosy. Journal of the Neurological Sciences, 420. https://doi.org/10.1016/j.jns.2020.117288

Lopes, G. L., Borges, M. G. L., Conceição, A. O., Batista, K. N. M., de Macedo, G. M. M., \& Xavier, M. B. (2017). Development and evolution of nerve damage in individuals with leprosy during medical treatment, at completion of MDT and after release from treatment. Indian Journal of Leprosy, 89(1).

Lowy L, \& Ridley DS. (1954). The acid-fast staining properties of Mycobacterium leprae. Transactions of the Royal Society of Tropical Medicine and Hygiene., 48, 406-410.

Ministério da Saúde. (2020). boletim-hanseniase-_-25-01.pdf.

Montalvão, L. M., Marques, G. L., Dhamer, D. S. V., Crepaldi, A. A., Sant'ana, A. P., \& Silva, L. (2018). Diagnóstico e tratamento da hanseníase: atuação do fisioterapeuta. Revista FAIPE, 8(1), 72-84.

Morimoto, A., Oh, Y., Nakamura, S., Shioda, Y., Hayase, T., Imamura, T., \& Imashuku, S. (2017). Inflammatory serum cytokines and chemokines increase associated with the disease extent in pediatric Langerhans cell histiocytosis. Cytokine, 97(December 2016), 73-79. https://doi.org/10.1016/j.cyto.2017.05.026

Mota, M. S. de A., Vilanova, V. A., Andrade, S. P., Silva, B. L. B., \& Ferreira, D. O. (2018). Ocorrência da hanseníase no Brasil e os principais métodos de diagnósticos laboratoriais. Scire Salutis, 7(2). https://doi.org/10.6008/spc2236-9600.2017.002.0002

Oliveira, J. M., Rêgo, J. L., de Lima Santana, N., Braz, M., Jamieson, S. E., Vieira, T. S., \& Castellucci, L. C. (2016). The -308 bp TNF gene polymorphism influences tumor necrosis factor expression in leprosy patients in Bahia State, Brazil. Infection, Genetics and Evolution, 39, 147-154. https://doi.org/10.1016/j.meegid.2016.01.026

Pereira, A. S., et al. (2018). Metodologia da pesquisa científica. [e-book]. Santa Maria. Ed. UAB/NTE/UFSM. https://repositorio.ufsm.br/bitstream/handle/1/15824/Lic_Computacao_Metodologia-Pesquisa-Cientifica.pdf?sequence=1.

Ridley, D. S., \& Jopling, W. H. (1966). Classification of leprosy according to immunity. A five-group system. International Journal Leprosy and Other Mycobacterium Disease, 34(3), 255-273. 
Research, Society and Development, v. 10, n. 6, e29010615823, 2021

(CC BY 4.0) | ISSN 2525-3409 | DOI: http://dx.doi.org/10.33448/rsd-v10i6.15823

Santana, E. M. F. de, Brito, K. K. G. de, Nogueira, J. D. A., Leadebal, O. D. C. P., Costa, M. M. L., Silva, M. A. da, \& Soares, M. J. G. O. (2018). Deficiências e incapacidades na hanseníase: do diagnóstico à alta por cura. Revista Eletrônica de Enfermagem, 20. https://doi.org/10.5216/ree.v20.50436

Souza, L. R. De, \& Peres, C. (2019). 1, 23 4, 16.

Sykam, A., Gutlapalli, V. R., Tenali, S. P., Meena, A. K., Chandran, P., Pratap, D. V. S., \& Suneetha, L. M. (2015). Association of tumor necrosis factor-alpha and interferon gamma gene polymorphisms and their plasma levels in leprosy, HIV and other peripheral neuropathies. Cytokine, 76(2), 473-479. https://doi.org/10.1016/j.cyto.2015.09.010

WHO. (1994). Chemotherapy of Leprosy. Geneva, World Health Organization. Technical Report Series., 847.

WHO. (2020). Weekly epidemiological record Relevé épidémiologique hebdomadaire, 2016(36), 417-440.Colocar espaço entre uma referência e outra. 